\title{
In Vitro Activity of Ro 23-9424 against Clinical Isolates of Legionella Species
}

\author{
PAUL H. EDELSTEIN ${ }^{1,2 *}$ AND MARTHA A. C. EDELSTEIN ${ }^{1}$ \\ Departments of Pathology and Laboratory Medicine ${ }^{1}$ and Medicine, ${ }^{2}$ University of \\ Pennsylvania School of Medicine, Philadelphia, Pennsylvania 19104-4283
}

Received 12 June 1992/Accepted 4 September 1992

\begin{abstract}
Agar and broth microdilution MICs of Ro 23-9424 that inhibited $90 \%$ of 22 Legionella clinical isolates tested were 0.64 and $0.08 \mu \mathrm{g} / \mathrm{ml}$, respectively; respective erythromycin values were 1.0 and $0.12 \mu \mathrm{g} / \mathrm{ml}$. Ro 23-9424 $(1 \mu \mathrm{g} / \mathrm{ml})$ was slightly more active than the same erythromycin concentration in a macrophage system, for both Legionella pneumophila strains studied.
\end{abstract}

Ro 23-9424 is a dual-action antibacterial agent in which desacetyl cefotaxime is covalently linked to fleroxacin (8). The drug is hydrolyzed to a limited extent in vivo and in vitro, with release of free fleroxacin $(2,8)$. Ro 23-9424 is broadly active against both gram-positive and some gramnegative bacteria $(1,9)$. It is unknown whether Ro 23-9424 is concentrated in cells. Fleroxacin, but not cefotaxime, is active for intracellular Legionella pneumophila and in a guinea pig model of $L$. pneumophila pneumonia $(6,13,14)$. We tested Legionella spp. with Ro 23-9424 by using a variety of susceptibility testing methods, designed to determine whether the drug is active for intracellular and extracellular Legionella spp. Two different extracellular susceptibility testing methods were used to allow comparison of results with those previously published by us and others using the two different methods (5-7, 10-13).

All legionellae studied were clinical isolates. These strains were identical to those used in prior studies and were composed of two strains each of $L$. dumoffi, $L$. longbeachae, and $L$. micdadei, one strain of $L$. bozemanii, and 15 strains of $L$. pneumophila $(5,7)$. Staphylococcus aureus ATCC 29213 and Escherichia coli ATCC 25922 were used as control organisms for susceptibility testing. Legionellae were grown on locally made buffered charcoal-yeast extract medium supplemented with $0.1 \% \alpha$-ketoglutarate (BCYE $\alpha$ ) (3). Incubation of all media was done at $35^{\circ} \mathrm{C}$ in humidified air. Standard powders of Ro 23-9424 and fleroxacin were obtained from Hoffmann-LaRoche, Inc., Nutley, N.J.; erythromycin powder was obtained from Abbott Laboratories, North Chicago, Ill.

Agar dilution susceptibility testing was performed as described previously (5). Briefly, antimicrobial agent-containing BCYE $\alpha$ agar plates were inoculated with $\approx 10^{5}$ CFU of bacteria. The control $S$. aureus and $E$. coli strains were inoculated onto antimicrobial agent-containing Mueller-Hinton agar plates, as well as BCYE $\alpha$ plates, to determine whether BCYE $\alpha$ medium inhibited antimicrobial agent activity. The plates were incubated for either $24 \mathrm{~h}$ (nonlegionellae) or $48 \mathrm{~h}$ (legionellae), at which time MICs were determined. Broth microdilution susceptibility testing was performed by using buffered yeast extract broth supplemented with $0.1 \% \alpha$-ketoglutarate (BYE $\alpha$ ) (legionellae) or with Mueller-Hinton broth (nonlegionellae), with a final volume of $200 \mu \mathrm{l}$ and a final bacterial concentration of $5 \times$

\footnotetext{
* Corresponding author.
}

$10^{5} \mathrm{CFU} / \mathrm{ml}$ (3). Otherwise, the microdilution method was performed exactly as described previously for a macrodilution method (5). All testing was done in duplicate, and results were expressed as geometric means. A MIC found to be less than or equal to the lowest antimicrobial agent concentration tested was arbitrarily defined to be the lowest concentration tested. Erythromycin was included as a control; data for the activity of this drug for the Legionella strains we tested, as measured by agar dilution susceptibility testing, have been presented previously (5).

Guinea pig pulmonary alveolar macrophages were harvested and purified as described previously (5). The final concentration of macrophages was approximately $10^{5}$ cells per well. Antimicrobial susceptibility testing of intracellular L. pneumophila was performed as described previously (5). Briefly, $\approx 10^{4}$ CFU of washed BCYE $\alpha$ plate-grown L. pneumophila was added to the purified alveolar macrophages. The bacteria and macrophages were incubated for 1 day after $1 \mathrm{~h}$ of incubation with shaking. Antimicrobial agents were added to the respective wells, after the wells had been washed three times to remove nonadherent bacteria. Sonic extracts of two replicate, non-antimicrobial agent-containing wells were quantitatively cultured for use as the day 1 bacterial count. Non-antimicrobial agent-containing wells were used as growth controls. After 2 more days of incubation, the supernatants were sampled and quantitatively cultured; all wells were then washed to remove antimicrobial agents. Bacterial counts in the supernatant of each well were determined for another 4 days. To check for antimicrobial agent toxicity, uninfected macrophages were incubated with the highest concentration of antimicrobial agent tested and observed microscopically daily. The relative activities of Ro 23-9424 and fleroxacin were determined for one $L$. pneumophila strain; the activity of fleroxacin alone $(0.5 \mu \mathrm{g} / \mathrm{ml})$ was contrasted to that of the combination drug $(1 \mu \mathrm{g} / \mathrm{ml})$. The fleroxacin extracellular broth microdilution MIC for $L$. pneumophila strain F2111 is $0.02 \mu \mathrm{g} / \mathrm{ml}$ (6). All experiments were carried out in triplicate, and quantitative plating was carried out in duplicate.

Ro 23-9424 and erythromycin agar and broth microdilution MICs for the 22 Legionella strains tested are shown in Table 1. A single $L$. micdadei strain did not grow well enough in BYE $\alpha$ broth for broth microdilution testing to be performed, allowing only 21 Legionella strains to be tested by this method. The erythromycin broth microdilution MIC for another $L$. micdadei strain $(0.5 \mu \mathrm{g} / \mathrm{ml})$ was much higher than that for any other strain tested. Erythromycin broth micro- 
TABLE 1. Agar and broth microdilution susceptibilities of 22 Legionella sp. strains

\begin{tabular}{|c|c|c|c|c|c|c|}
\hline \multirow{3}{*}{ Drug } & \multicolumn{6}{|c|}{ MIC ( $\mu \mathrm{g} / \mathrm{ml})$ with: } \\
\hline & \multicolumn{3}{|c|}{ BCYE $\alpha$ agar } & \multicolumn{3}{|c|}{ BYE $\alpha$ broth } \\
\hline & $\mathrm{MIC}_{50}$ & $\mathrm{MIC}_{90}$ & Range & $\mathrm{MIC}_{50}$ & $\mathrm{MIC}_{90}$ & Range \\
\hline Ro 23-9424 & 0.64 & 0.64 & 0.16-1.28 & 0.08 & 0.08 & $\begin{array}{r}0.04-0.08 \\
<0.06-0.5\end{array}$ \\
\hline Erythromycin & 0.12 & 1.0 & $\leq 0.06-1.0$ & 0.06 & 0.12 & $\leq 0.06-0.5$ \\
\hline
\end{tabular}

dilution MICs for strains F889 and F2111 were $\leq 0.06$ and $0.125 \mu \mathrm{g} / \mathrm{ml}$, respectively. Ro $23-9424$ broth microdilution MICs for the same strains were $0.08 \mu \mathrm{g} / \mathrm{ml}$.

Erythromycin, but not Ro 23-9424, was slightly inhibited by BCYE $\alpha$ agar, as determined by its MIC for the control $S$. aureus strain by using BCYEa and Mueller-Hinton agar media. The erythromycin MIC for $S$. aureus was $1 \log _{2}$ dilution greater with BCYE $\alpha$ agar. However, BCYE $\alpha$ agar inhibition of Ro 23-9424 was observed when MICs for $E$. coli were determined; the Ro 23-9424 MIC for the $E$. coli control strain was $2 \log _{2}$ dilutions greater with BCYE $\alpha$ agar than with Mueller-Hinton agar. Ro 23-9424 and erythromycin MICs for the non-Legionella sp. control strains were both 1 $\log _{2}$ dilution greater with BYE $\alpha$ broth than with MuellerHinton broth.

The major differences in MICs with agar and broth dilutions that were detected in this study have been noted by us and others previously $(5-7,11)$. Many feel that use of non-charcoal-containing test media gives the most accurate results because of decreased antibiotic inhibition by these media, in comparison to results obtained by using BCYE $\alpha$ medium. However, there exist no clinical data which would determine which method is more accurate. The additional complication of sometimes very poor correlation between in vitro susceptibility of $L$. pneumophila and results of clinical and animal studies makes such in vitro testing only a very rough guide to potential clinical utility of antimicrobial agents for the treatment of Legionnaires' disease (reviewed in reference 4). No previous studies of the activity of Ro 23-9424 against Legionella spp. have been done, making comparison of these results with those of other studies impossible. Both cefotaxime and fleroxacin alone are active against extracellular $L$. pneumophila, with fleroxacin MICs for $90 \%$ of strains tested $\left(\mathrm{MIC}_{90} \mathrm{~s}\right)$ of 0.64 and $0.04 \mu \mathrm{g} / \mathrm{ml}$ by agar and broth microdilutions, respectively, for the same strains used in this study (6) and cefotaxime $\mathrm{MIC}_{90} \mathrm{~s}$ of 4 and $\leq 0.12 \mu \mathrm{g} / \mathrm{ml}$ by agar and broth microdilutions, respectively, for different $L$. pneumophila strains $(10,12)$. Since we did not test equimolar concentrations of the two Ro 23-9424 components, it is unknown how similar the activity of the combination drug is to that of the combined free components.

The intracellular activity of Ro 23-9424 and erythromycin for two $L$. pneumophila serogroup 1 strains grown in guinea pig alveolar macrophages is shown in Fig. 1 and 2. For $L$. pneumophila strain F889 (Fig. 1), both drugs were only inhibitory in their activity, although there was a significantly longer postantibiotic effect observed for Ro 23-9424. Both erythromycin and Ro 23-9424 were similar in their inhibitory activity for L. pneumophila F2111 (Fig. 2). Fleroxacin alone $(0.5 \mu \mathrm{g} / \mathrm{ml})$ was more active than Ro $23-9424(1.0 \mu \mathrm{g} / \mathrm{ml})$, although the fleroxacin concentration was the same in both the single and combination drugs. The slight decrease in viable counts of strain F2111 observed on day 5, for eryth-

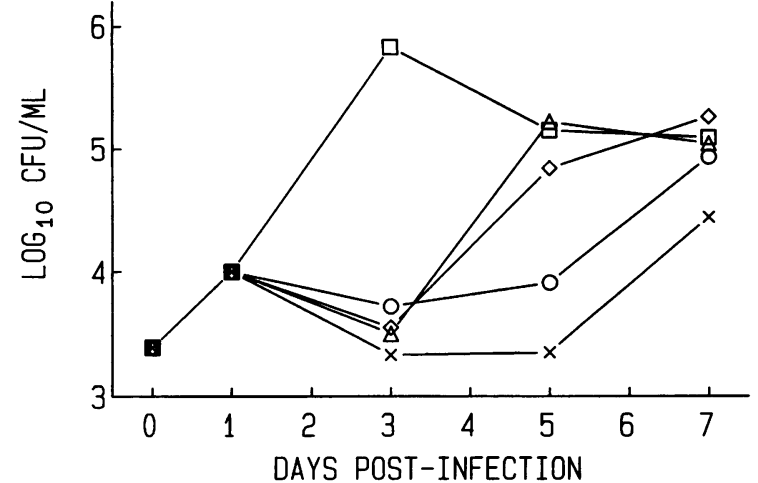

FIG. 1. Growth of $L$. pneumophila F889 serogroup 1 in guinea pig alveolar macrophages at postinfection days 1 to 7 . Symbols: $\square$, growth control; $\triangle$ and $\diamond, 0.25$ and $1.0 \mu \mathrm{g}$ of erythromycin per $\mathrm{ml}$, respectively; $O$ and $\times, 0.25$ and $1.0 \mu \mathrm{g}$ of Ro 23-9424 per ml, respectively.

romycin and Ro 23-9424 (both $0.25 \mu \mathrm{g} / \mathrm{ml}$ ), is artifactual because of macrophage killing by the bacteria by day 3 ; many of the bacteria in these tissue culture wells were washed out with the damaged macrophages. Macrophage toxicity caused by antimicrobial agents alone was not observed.

These studies show that Ro 23-9424 is about as active as erythromycin for both extracellular and intracellular Legionella spp., with neither drug being bactericidal. The significantly greater activity of fleroxacin than that of the combination drug is probably related to limited extracellular hydrolysis of the combination drug, which releases free fleroxacin. The free fleroxacin is probably more concentrated in macrophages than is the combination drug. Because L. pneumophila is protected from extracellular antimicrobial agents by its intracellular location in the lungs and elsewhere in the body, the in vitro activity of drugs against intracellular L. pneumophila is generally a good predictor of their clinical activity. Ro 23-9424 has potential use for the treatment of Legionnaires' disease, and possibly other intracellular infections, pending animal model treatment studies and clinical trials. Whether the combination drug would be more or less

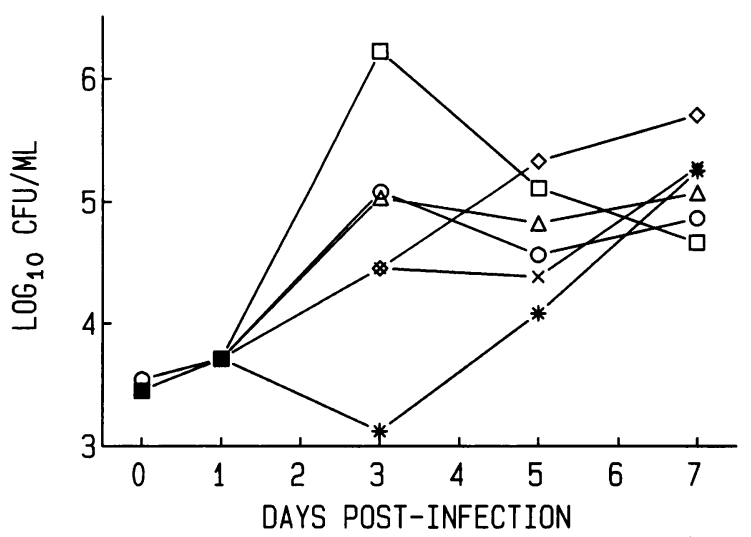

FIG. 2. Growth of $L$. pneumophila F2111 serogroup 1 in guinea pig alveolar macrophages at postinfection days 1 to 7. Symbols: $\square$, growth control; $\Delta$ and $\diamond, 0.25$ and $1.0 \mu \mathrm{g}$ of erythromycin per $\mathrm{ml}$, respectively; $O$ and $x, 0.25$ and $1.0 \mu \mathrm{g}$ of Ro $23-9424$ per ml, respectively; *, $0.5 \mu \mathrm{g}$ of fleroxacin per $\mathrm{ml}$. 
clinically effective than erythromycin can only be determined by comparative clinical studies.

This study was funded in part by Hoffmann-LaRoche, Inc. Minxia Liu provided excellent technical assistance.

\section{REFERENCES}

1. Beskid, G., V. Fallat, E. R. Lipschitz, D. H. McGarry, R. Cleeland, K. Chan, D. D. Keith, and J. Unowsky. 1989. In vitro activities of a dual-action antibacterial agent, Ro 23-9424, and comparative agents. Antimicrob. Agents Chemother. 33:1072 1077.

2. Christenson, J. G., K. K. Chan, R. Cleeland, B. Dix-Holzknecht, H. H. Farrish, Jr., I. H. Patel, and A. Specian. 1990. Pharmacokinetics of Ro 23-9424, a dual-action cephalosporin, in animals. Antimicrob. Agents Chemother. 34:1895-1900.

3. Edelstein, P. H. 1985. Legionnaires' disease laboratory manual, 3rd ed. Document no. 86-129871. National Technical Information Service, Springfield, Va.

4. Edelstein, P. H. In D. Raoult (ed.), Antimicrobial agents and intracellular pathogens, in press. CRC Press Inc., Boca Raton, Fla.

5. Edelstein, P. H., and M. A. C. Edelstein. 1989. WIN 57273 is bactericidal for Legionella pneumophila grown in alveolar macrophages. Antimicrob. Agents Chemother. 33:2132-2136.

6. Edelstein, P. H., M. A. C. Edelstein, and B. Holzknecht. 1992. In vitro activities of fleroxacin against clinical isolates of Legionella spp., its pharmacokinetics in guinea pigs, and use to treat guinea pigs with $L$. pneumophila pneumonia. Antimicrob. Agents Chemother. 36:2387-2391.
7. Edelstein, P. H., M. A. C. Edelstein, J. Weidenfeld, and M. B. Dorr. 1990. In vitro activity of sparfloxacin (CI-978; AT-4140) for clinical Legionella isolates, pharmacokinetics in guinea pigs, and use to treat guinea pigs with $L$. pneumophila pneumonia. Antimicrob. Agents Chemother. 34:2122-2127.

8. Georgopapadakou, N. H., A. Bertasso, K. K. Chan, J. S. Chapman, R. Cleeland, L. M. Cummings, B. A. Dix, and D. D. Keith. 1989. Mode of action of the dual-action cephalosporin Ro 23-9424. Antimicrob. Agents Chemother. 33:1067-1071.

9. Gu, J., and H. C. Neu. 1990 . In vitro activity of Ro 23-9424, a dual-action cephalosporin, compared with activities of other antibiotics. Antimicrob. Agents Chemother. 34:189-195.

10. Jones, R. N., and A. L. Barry. 1987. Antimicrobial activity of imipenem and SCH34343 against Legionella species. Diagn. Microbiol. Infect. Dis. 6:307-309.

11. Liebers, D. M., A. L. Baltch, R. P. Smith, M. C. Hammer, and J. V. Conroy. 1989. Susceptibility of Legionella pneumophila to eight antimicrobial agents including four macrolides under different assay conditions. J. Antimicrob. Chemother. 23:37-41.

12. Ruckdeschel, G., W. Ehret, and A. Ahl. 1984. Susceptibility of Legionella species to imipenem and 27 other beta-lactam antibiotics. Eur. J. Clin. Microbiol. 3:463-467.

13. Toro, J., K. Sawatari, T. Kanda, A. Saito, and K. Hara. 1983. New B-lactamase-resistant cephem treatment of guinea pigs infected with Legionella pneumophila. Microbiol. Immunol. 27:649-654.

14. Yoshida, S., and Y. Mizuguchi. 1984. Antibiotic susceptibility of Legionella pneumophila Philadelphia-1 in cultured guinea-pig peritoneal macrophages. J. Gen. Microbiol. 130:901-906. 